\title{
Das Jahrhundert der Stichproben
}

\author{
Andreas Quatember \\ Johannes Kepler Universität, Linz
}

\begin{abstract}
Zusammenfassung: Die Stichprobenmethode erfreut sich in der Gegenwart allgemeiner Akzeptanz und immer neue Wissenschaftsbereiche bedienen sich ihrer auf der Suche nach „Wahrheit“. Der Weg zu dieser Anerkennung war mühsam von den spärlichen, eher intuitiv begründeten Anfängen bis zur vollständigen Entwicklung der Theorie im 20. Jahrhundert. In diesem Aufsatz wird dieser Weg der Stichprobentheorie und -praxis bis in die Gegenwart mit Berücksichtigung des deutschprachigen Raums nachgezeichnet. Die Darstellung lässt als Folgerung zu, vom 20. Jahrhundert auch als dem Jahrhundert der Stichproben zu sprechen.
\end{abstract}

\begin{abstract}
The sampling method is commonly accepted in the present and always new areas of science use it in their search for the "truth". The way to this level of acceptance was laborious from the sparsely, rather intuitively founded beginning to the complete development of the theory in the 20th century. In this paper this way of the sampling theory and practice until the present is portrayed with consideration of the germanspeaking Europe. The description allows the conclusion, that from the 20 th century can also be spoken as the century of samples.
\end{abstract}

Schlüsselwörter: Stichprobentheorie, Stichprobenpraxis, Geschichte.

\section{$1 \quad$ Einleitung}

Wir sehen, schmecken, hören oder riechen nur Ausschnitte unserer (Um-) Welt. Das unbewusste Schlussfolgern von unseren Beobachtungen auf die damit mehr oder weniger unvollständig beschriebene Wirklichkeit ist Bestandteil des menschlichen Alltags und in seiner Urform vermutlich als Überlebensstrategie entstanden.

Das im Gegensatz dazu bewusste Schlussfolgern von einer Teilmenge auf die Gesamtheit wird als Stichprobenmethode bezeichnet. Auch dafür gibt es Beispiele aus unserem Alltag: Wir bedienen uns dieser Methode immer, wenn wir Speisen abschmecken, Weine verkosten oder Parfüms testen. Auch beim Ablegen von Prüfungen hat jeder von uns schon Erfahrungen mit der Stichprobenmethode gesammelt.

Für den wahrscheinlichkeitstheoretischen Schluss vom Ergebnis einer solchen Stichprobe auf die ihr zu Grunde liegende Grundgesamtheit werden Verfahren verwendet, welche die konkrete Auswahl der für diese Folgerung benötigten Untersuchungsobjekte aus der Grundgesamtheit ganz genau regeln. Die Entwicklung solcher „Stichprobenverfahren“ und die Untersuchung der statistischen Eigenschaften der damit zusammenhängenden Schätzer für unbekannte Parameter sind Gegenstand der statistischen Stichprobentheorie.

Während nun aber die alltäglichen unbewussten Stichproben zweifellos seit jeher ein charakteristischer Bestandteil (nicht nur) des menschlichen Lebens sind, sind institutionalisierte bewusste Stichproben aus der Zeit vor der theoretischen Auseinan- 
dersetzung mit der Stichprobenmethode nur sehr spärlich belegt. Die Stichprobentheorie selbst ist schließlich vollständig im 20. Jahrhundert entwickelt worden.

\section{2 „Prätheoretische“ Stichproben}

Aus dem Altertum sind nur vereinzelte Anwendungen der Stichprobenmethode bekannt. Eine der ersten belegten, systematischen Anwendungen ist die unter König Eduard I. im England des 13. Jahrhunderts eingeführte Gewichtsprüfung der in der königlichen Münze zu London geprägten Gold- und Silbermünzen (vgl. Stigler, 1977). Dabei wurden über einen gewissen Zeitraum Teile der täglichen Münzproduktion in einem Behälter gesammelt, um die Einhaltung des zwischen der Münze und dem König vertraglich vereinbarten Münzgewichtes überprüfen zu können. Für die Kontrolle wurde dieser „Büchse“ nach einem nicht näher beschriebenen Verfahren abermals ein Teil ihres Inhalts entnommen und erhoben, ob dessen Gesamtgewicht innerhalb der vereinbarten Toleranzgrenzen liegt.

Als der Astronom Edmond Halley im Jahr 1693 die Daten über Geburtenziffern und Todesfälle der Jahre 1687 bis 1691 in der Stadt Breslau erhielt, fand die Stichprobenmethode Anwendung auf statistische Sterblichkeitsprobleme. Im Gegensatz zu den großen britischen Städten besaß die Bevölkerung von Breslau eine relativ geringe Fluktuation. Dieser Umstand diente Halley in seinem Aufsatz: „An estimate of the degrees of the mortality of mankind, drawn from curious tables of the births and funerals at the city of Breslaw; with an attempt to ascertain the price of annuities upon lives“ als Begründung dafür, dass er die Absterbeordnung der Einwohner Breslaus und nicht einer britischen Stadt zur Berechnung von Prämien für Lebensversicherungen in Großbritannien heranzog (vgl. Halley, 1693, S. 202).

Der schottische Arzt John Arbuthnott überprüfte zu Beginn des 18. Jahrhunderts durch die Aufzeichnungen über die Geschlechtsverteilung der Geburten in London in 82 aufeinanderfolgenden Jahren die Hypothese, dass gleich viele Mädchen wie Knaben geboren werden und kam zu dem Schluss, dass dies nicht der Fall sein kann (vgl. Arbuthnott, 1710). Er interpretierte diese Erkenntnis im Übrigen als Nachweis der Existenz Gottes.

Weitere „prätheoretische“ Schlussfolgerungen von einem bekannten Teil auf das unbekannte Ganze hatten Schätzungen der Gesamtbevölkerungszahlen zum Gegenstand: Sir Frederick Morton Eden errechnete, nachdem schon in der 2. Hälfte des 18. Jahrhunderts vage Bevölkerungsschätzungen in Großbritannien versucht worden waren, im Jahre 1800 die Bevölkerungsziffer durch Benützung von Stichprobenergebnissen über die durchschnittliche Anzahl von Hausbewohnern und über die Anzahl von Geburten. Die erste Volkszählung in Großbritannien im Jahre 1801 bestätigte die Güte seiner damit gewonnenen Schätzung (vgl. Stephan, 1948).

Bemerkenswerterweise ging also die Stichprobenmethode der Vollerhebung in der amtlichen Statistik voraus. Aus Frankreich sind Versuche auf diesem Gebiet ebenfalls schon seit dem ausklingenden 18. Jahrhundert überliefert. Der Mathematiker Pierre Simon de Laplace führte seine Berechnungen im Jahre 1802 durch. Er versuchte auch eine erste grobe Bestimmung der Genauigkeit seiner Schätzung (ebd., S. 15). 
Etwa um dieselbe Zeit wurden in England auch die ersten Untersuchungen der Lebensumstände der neu entstandenen Schicht der Arbeiterschaft durchgeführt. 1797 verfasste etwa Eden einen Bericht mit dem Titel: „Der Staat der Armen oder die Geschichte der arbeitenden Klassen in England, von der Eroberung bis zur Gegenwart unter besonderer Berücksichtigung ihrer häuslichen Verhältnisse; in Bezug auf Nahrungsmittel, Kleidung, Heizmaterial und Wohnung usw.". Dafür standen als Daten die von den örtlichen Pfarreien gesammelten Haushaltsrechnungen einiger Landarbeiterfamilien zur Verfügung (vgl. Kenessey, 1964, S. 315). Auch außerhalb Großbritanniens führte die beginnende Industrialisierung in Europa zu sozialen Veränderungen, die solche Untersuchungen erforderlich machten. Da dafür einzelne Fälle umfassend untersucht wurden, spricht man in diesem Zusammenhang von „Fallstudien“.

In den U.S.A. starteten in der Mitte des 19. Jahrhunderts auch landwirtschaftliche Stichprobenerhebungen mit freiwilligen Teilnehmern zur Schätzung z.B. des Ernteertrages oder des Viehbestandes. Auch Wirtschaftsstatistiken, die Auskunft über Preise, Löhne, Arbeitszeit, Beschäftigung und Produktion geben sollten, wurden auf diese Weise bestimmt. Ebenfalls zu dieser Zeit begann in den U.S.A. die Erforschung der „öffentlichen Meinung“ mit Probeabstimmungen zur Vorhersage von Wahlergebnissen. Dabei wurden anfänglich Stimmzettel in Zeitungen abgedruckt, die von den Lesern an die Redaktionen zurückgesandt werden konnten. Auch die öffentliche Wirkung von Politikern war schon bald ein Thema für Stichprobenerhebungen.

Bei all diesen Anwendungen der Stichprobenmethode wurden die Belange der Auswahl der Untersuchungsobjekte für die Stichprobe noch sehr vernachlässigt. Jede beliebige Teilmenge des Ganzen wurde für die Verallgemeinerung der Stichprobenergebnisse auf die Grundgesamtheit als geeignet betrachtet.

Die amtliche Statistik stützte sich dagegen ab dem Beginn des 19. Jahrhunderts ausschließlich auf Vollerhebungen der Bevölkerungen. Diese wurden von den amtlichen Statistikern als einzige Möglichkeit erachtet, die benötigten zuverlässigen Informationen über die betreffenden Grundgesamtheiten zu erhalten. Der Leiter des norwegischen statistischen Bundesamtes in den Jahren 1867 bis 1913, Anders N. Kiaer, wurde als Verantwortlicher für den im Zehnjahresrhythmus durchzuführenden Zensus in Norwegen angesichts des außerordentlich hohen Aufwandes der Volkszählungen und der lange dauernden Auswertung der damit erhobenen Daten aktiv (vgl. Seng, 1951, S. 214ff; Kruskal und Mosteller, 1980, S. 174ff). Er ließ als Ergänzung zu dieser Vollerhebung 1894 erstmals Teilerhebungen der norwegischen Bevölkerung durchführen.

Im darauffolgenden Jahr stellte er beim Kongress des „International Statistical Institut (ISI) " in Bern sein Konzept der Auswahl der Untersuchungsobjekte für diese Teilerhebung der Grundgesamtheit, die „repräsentative Methode“, dem anwesenden Fachpublikum vor. Zu den bisher üblichen Vorgangsweisen stellte er fest, „daß bei diesen höchst interessanten Untersuchungen die Stichprobenmethode als solche nicht genügend geprüft worden war" (zitiert nach Kenessey, 1964, S. 319). Seine Vorgangsweise sollte eine Verallgemeinerung der Stichprobenergebnisse durch eine bewusst herbeigeführte Ähnlichkeit von Stichprobe und Grundgesamtheit 
gewährleisten. Diese Forderung sollte dadurch eingehalten werden, dass der für die Stichprobe ausgewählte Teil der Bevölkerung hinsichtlich der beruflichen Zusammensetzung den Verhältnissen in der Grundgesamtheit entspricht.

Kiaers Zugang zur Stichprobenthematik war dabei rein intuitiv und nicht theoretisch fundiert. Seine Ideen stießen im Rahmen des Kongresses auch auf schärfsten Widerspruch: Einer seiner schärfsten Kritiker war der bayerische Universitätsstatistiker Georg von Mayr. Er betonte, dass man trotz mancher Einsatzmöglichkeit für die Stichprobenmethode niemals darauf vergessen dürfe, dass eine Stichprobe eine Vollerhebung nicht ersetzen kann: „Keine Berechnungen, wenn Beobachtungen gemacht werden können" (zitiert nach Kruskal und Mosteller, 1980, S. 175; Übersetzung durch den Verf.). Andere Teilnehmer des Kongresses wollten die Diskussion über Kiaers Thematik überhaupt wegen deren vermeintlicher Wertlosigkeit abbrechen.

Kiaer aber ließ sich von diesen Widersprüchen nicht entmutigen und unternahm schon beim darauffolgenden Kongress des ISI 1897 in St. Petersburg einen neuerlichen Vorstoß. Er hatte seine Vorschläge inzwischen konkretisiert. Zur bildlichen Darstellung seiner Idee entwickelte er die „Zwei-Fässer-Analogie“: Man stelle sich ein kleines und ein großes Fass vor. Wenn Stichproben von beiden Fässern ähnliche Inhalte aufweisen, dann darf mit den Ergebnissen der Analyse des kleinen Fasses auf das große geschlossen werden. Ferner benutzte er zur deutlichen Abgrenzung gegenüber den Fallstudien das Symbol einer Fotografie, welche die Details des Originals in den richtigen Proportionen wiedergibt, und er betonte, dass Fallstudien kein solches Abbild der Gesamtheit erzeugen. Bei seinem Vortrag deutete er auch die Möglichkeit an, die „Repräsentativität“ einer Stichprobe an Hand der Übereinstimmung der Verteilung der Stichprobe auf bestimmten Merkmalen, für die Volkszählungsergebnisse vorhanden sind, zu kontrollieren. Im Falle ausreichender Übereinstimmung bei diesen Vergleichen soll daraus gefolgert werden können, dass dies auch für die nicht kontrollierbaren Merkmale gilt. Schließlich kam er zur Überzeugung, dass der wissenschaftliche Wert von Teilerhebungen mehr von diesem repräsentativen Charakter der Stichprobe als von den Stichprobenumfängen abhängt. Das ISI setzte in der Folge einen Ausschuss ein, der die Methode eingehend diskutieren und für den nächsten Kongress einen Bericht verfassen sollte.

Dieses nächste Treffen des Institutes fand 1901 in Budapest statt. Kiaer konnte mit Berichten über einige in Norwegen durchgeführte Stichprobenuntersuchungen die Bemühungen um die Akzeptierung seiner Idee fortsetzen. Caroll D. Wright unterstützte Kiaer in Budapest mit einem Beitrag über die positiven Erfahrungen, die in der amtlichen Statistik der Vereinigten Staaten ebenfalls bereits mit der Stichprobenmethode gemacht wurden. Neben der amerikanischen amtlichen Statistik bediente sich auch die indische bald der Stichprobenmethode. P.C. Mahalanobis und seine Kollegen entwarfen dabei Verfahren, die sie hauptsächlich für die Ernteschätzung in der Landwirtschaft und zur Erhebung der sozialen Lage der indischen Bevölkerung einsetzten (vgl. Kenessey, 1964, S. 325).

Als besonders nützlich erwies sich die Stichprobenmethode natürlich für die amtliche Statistik im riesigen russischen Zarenreich, wo sie schon mit Beginn der 2. Hälfte des 19. Jahrhunderts Anwendung fand und später die Kiaerschen Ideen 
durch A.I. Tschuprow verbreitet wurden, wodurch der Keim für eine regelrechte Schule der Stichprobentheorie in Russland gelegt wurde (vgl. ebd., S. 322).

In der in Budapest stattfindenden Diskussion formulierte der in St. Petersburg geborene Statistiker und Ökonom Ladislaus von Bortkiewicz eine Idee zur Kontrolle der Repräsentativität einer Stichprobe: Anstatt durch die Differenz zwischen Stichprobenergebnissen und Zensusdaten abzuschätzen - wie dies Kiaer 1897 vorschlug -, ob die für die Verallgemeinerbarkeit der Stichprobenergebnisse nötige Übereinstimmung von Stichprobe und Grundgesamtheit in ausreichendem Maße gewährleistet ist, soll eine Wahrscheinlichkeit berechnet werden, die angibt, ob die gefundene Abweichung als zufällig erachtet wird oder nicht. Fällt diese Wahrscheinlichkeit gering aus, dann dürfen die Stichprobenergebnisse auch für die nicht überprüfbaren Merkmale nicht als repräsentativ für die Grundgesamtheit gelten.

Von Bortkiewicz folgte damit gedanklich den Spuren Karl Pearsons, der sich am Ende des 19. Jahrhunderts mit dem Problem der Anpassung beobachteter Verteilungen an theoretische beschäftigte. Im Jahre 1900 stellte er in seinem Aufsatz „On the Criterion that a Given System of Deviations from the Probable in the Case of a correlated System of Variables is Such that it Can be Reasonably Supposed to have Arisen from Random Sampling“ den $\chi^{2}$-Anpassungstest vor.

Beim darauffolgenden Kongress 1903 in Berlin trugen Kiaers Bemühungen erstmals Früchte, als der 6 Jahre zuvor eingesetzte Ausschuss in seinem Bericht endlich die Verwendung der „repräsentativen Methode“ für bestimmte Fälle empfahl. Voraussetzung für die Verwendung sollte allerdings sein, dass bei der Publikation der Stichprobenergebnisse der Auswahlvorgang vollständig beschrieben wird!

Der französische Statistiker Lucien March nahm das durch von Bortkiewicz zwei Jahre zuvor diskutierte Thema erneut auf und stellte fest, dass für die Repräsentativität der Stichprobe hinsichtlich der Untersuchungsmerkmale die zufällige Ziehung der Untersuchungsobjekte aus der Grundgesamtheit vorausgesetzt werden muss. Für diese zufällige Ziehung schlug er - ohne diese Begriffe schon zu verwenden - die uneingeschränkte Zufallsauswahl und das Klumpenverfahren vor und veranschaulichte diese Vorgangsweisen mit Hilfe des vom holländischen Physiker Christian Huygens schon 250 Jahre zuvor zur Beschreibung seiner wahrscheinlichkeitstheoretischen Überlegungen entwickelten Urnenmodells (vgl. Kruskal und Mosteller, 1980, S. 177f).

\section{Das Jahrhundert der Stichprobentheorie und -praxis}

Was Lucien March 1903 in Berlin gedanklich vorbereitete, wurde schließlich vom britischen Mathematiker Arthur L. Bowley theoretisch ausgeführt. Bowley war ein herausragender Praktiker, der große Untersuchungen in den Bereichen der ökonomischen und der Sozialstatistik durchführte (vgl. Maunder, 1972). Aber auch seine theoretischen Arbeiten hatten größte Bedeutung für die Stichprobentheorie. In seinem im Jahr 1906 im Journal of the Royal Statistical Society veröffentlichten Beitrag „Address to the Economic and Statistics Section of the British Association for the 
Advancement of Science“ thematisierte er die Technik der „repräsentativen Methode“ und er wies darauf hin, dass man „so gute Resultate, wie man es nur wünscht, durch Stichproben erhalten kann und sehr häufig wirklich kleine Stichproben ausreichen" (zitiert nach Bellhouse, 1988, S.5).

Er suchte in diesem Aufsatz nach einer empirischen Bestätigung für die Anwendung des Zentralen Grenzwertsatzes bei der Verteilung von Mittelwertschätzern in uneingeschränkten Zufallsauswahlen. Zur Überprüfung dieser Hypothese zog Bowley unter Verwendung eines groben Ersatzes für Zufallszahlen (er verwendete die letzten Stellen in einer Tabelle des Nautical Almanac) eine uneingeschränkte Zufallsauswahl von Zinsraten vom Umfang $n=400$ aus einer Grundgesamtheit von $N=3.878$ Anleihen. Aus dieser Stichprobe bildete er in der Reihenfolge der Ziehung ihrer Elemente 40 Gruppen zu je 10 Zinsraten und verglich schließlich die empirische Stichprobenverteilung der 40 Gruppenmittelwerte mit einer Normalverteilung. Das Ergebnis interpretierte er als gute Übereinstimmung zwischen Empirie und Theorie.

Seine Schlussfolgerung, dass der Erwartungswert und die Varianz dieser Stichprobenverteilung unabhängig von der Größe der Grundgesamtheit sind, erwies sich jedoch nicht als völlig zutreffend: 12 Jahre später ergänzte L. Isserlis nämlich Bowleys Varianzdarstellung durch die Endlichkeitskorrektur (vgl. Bellhouse, 1988, S. 4ff). Bowleys Arbeit aus dem Jahr 1906 aber markierte den Eintritt der Wahrscheinlichkeitstheorie in die Geschichte der Stichproben und sie begründete damit die Stichprobentheorie.

Seine bedeutendste praktische Arbeit war die große Armutsstudie in Reading im Jahr 1912. Für die Untersuchung der Lebensverhältnisse in dieser britischen Kleinstadt zog Bowley eine systematische Auswahl von Gebäuden aus vorhandenen Listen (jedes zehnte Gebäude). Systematische Auswahlen waren als Stichprobenverfahren schon einige Jahre z.B. in der Forstwirtschaft in Gebrauch (vgl. Stephan, 1948, S.21f). In seinem Bericht über die Ergebnisse dieser Untersuchung wendete Bowley die Resultate seiner für uneingeschränkte Zufallsauswahlen entwickelten theoretischen Überlegungen an. Erst später bemerkte er den möglichen Unterschied zwischen uneingeschränkten Zufallsauswahlen und systematischen Auswahlen aus der Grundgesamtheit (vgl. Bellhouse, 1988, S. 6).

Bowley benutzte das als Kennzeichen uneingeschränkter Zufallsauswahlen formulierte Prinzip der für alle Objekte gleichen Auswahlwahrscheinlichkeit in der Praxis auch in geschichteten und geklumpten Zufallsauswahlen. Dass damit auch die Geburtsstunde für die theoretische Auseinandersetzung mit diesen Stichprobenverfahren läutete, unterstreicht Bowleys Einfluss auf die weitere Entwicklung der Stichprobentheorie.

Inzwischen beschäftigte sich Ronald A. Fisher, Statistiker an der landwirtschaftlichen Versuchsstation von Rothamsted in England, mit einer unabhängig von der Kenntnis der a-priori-Verteilung aufgebauten Schätztheorie. Sein Aufsatz mit dem Titel „On the Mathematical Foundations of Theoretical Statistics“ aus dem Jahre 1922, in dem dieses Konzept formuliert wurde, beinhaltete auch erstmals die Gütekriterien der Konsistenz, Effizienz und Suffizienz eines Schätzers und stellte die Maximum-Likelihood-Methode für die Bestimmung von Schätzern vor, die diese Kriterien aufweisen (vgl. Fisher, 1922). Auch dies hatte große Bedeutung für die Ausbreitung 
Tabelle 1: Die Dokumentation von Qualitätskriterien für Umfragen in Zeitungsartikeln (vgl. Kaase, 1999, S. 77).

\begin{tabular}{lc}
\hline Information & relative Häufigkeit \\
\hline Name des Umfrageinstituts & 0,98 \\
Grundgesamtheit & 0,72 \\
Zeitraum der Befragung & 0,63 \\
Stichprobenumfang & 0,59 \\
Auftraggeber & 0,57 \\
Stichprobenverfahren & 0,52 \\
Fragewortlaut & 0,33 \\
Stichprobenfehler & 0,03 \\
\hline
\end{tabular}

der Stichprobenmethode.

Nach einer Zeitspanne von nahezu 20 Jahren wandte sich 1924 das ISI mit der Einsetzung eines Ausschusses, der die Anwendungen der Stichprobenmethoden der letzten Jahre untersuchen sollte, wieder der Stichprobenthematik zu. Bowley war ein maßgebliches Mitglied dieses Gremiums, das bei der folgenden Tagung des ISI 1925 in Rom seinen Bericht vorlegte. Auf diesem basierend verabschiedete das ISI eine Resolution, die endlich auf die bedeutenden Vorteile hinwies, welche die Verwendung der Stichprobenmethode unter bestimmten Voraussetzungen gegenüber einer Vollerhebung aufweist.

Für die Ziehung der Stichprobe wurden zwei Verfahren akzeptiert: Eine Zufallsauswahl mit gleichen Auswahlwahrscheinlichkeiten für alle Objekte der Grundgesamtheit (dazu zählte neben der uneingeschränkten auch die von Bowley in der Praxis bereits angewendete geschichtete Zufallsauswahl mit proportionaler Aufteilung des Stichprobenumfanges auf die Schichten) und eine bewusste Auswahl von Gruppen von Untersuchungsobjekten. Das ISI empfahl in seiner Resolution weiters, die Stichproben so zu ziehen, dass eine mathematische Angabe der Genauigkeit der Resultate erfolgen kann. Und schließlich wurde - wie schon in der Resolution des Jahres 1903 - der Wunsch geäußert, dass der Bericht über die Ergebnisse einer Stichprobe eine detaillierte Beschreibung des verwendeten Stichprobenverfahrens enthalten soll.

Dies ist eine seither wiederholt geäußerte und auch gegenwärtig noch oft unerfüllte Forderung. Brettschneider untersuchte z.B. 1996 die über Wahlumfragen zu den Bundestagswahlen von 1980 bis 1994 in vier deutschen Qualitätszeitungen erschienenen Artikel auf die Anwesenheit von acht verschiedenen die Güteeinschätzung der Stichprobenergebnisse ermöglichenden Informationen (Tabelle 1) (zitiert in Kaase, 1999, S. 77).

Die beiden in der Resolution von 1925 beschriebenen Stichprobenverfahren blieben für ein Jahrzehnt die für Schlussfolgerungen von Stichproben auf Grundgesamtheiten institutionell akzeptierten Auswahlmethoden. Bowley fasste seine Erkenntnisse zu den beiden Auswahlmethoden 1926 in einer Monografie zusammen (vgl. Bellhouse, 1988, S. 6).

Bowleys Versuch der Realisierung einer Zufallsauswahl durch Verwendung von 
Zahlen aus einem nautischen Tabellenwerk im Jahr 1906 war eine der frühesten in der Stichprobentheorie angewandten Techniken der praktischen Umsetzung des Zufallskonzepts. Zwei Jahre später schrieb W.S. Gosset, Chemiker der Guiness-Brauerei, für die Überprüfung seiner t-Verteilung Messwerte von 3.000 Kriminellen auf Kartons und zog nach einem Mischvorgang alle Kartons ohne Zurücklegen (vgl. Student, 1908, S. 47). Um 1910 wurde im schwedischen Göteborg für eine Wohnungsstudie eine zufällige Auswahl aus Adressenkarten gezogen (vgl. Dalenius, 1957, zitiert in Kruskal und Mosteller, 1980, S. 179). Diese Beispiele „physischer Randomisierung“ blieben jedoch Einzelfälle. Die Regel war eine systematische bzw. eine bewusste Auswahl aus der Grundgesamtheit. Erst die Publikation von Zufallszahlentabellen im Jahre 1927 durch L.H.C. Tippett in der Zeitschrift Tracts for Computers förderte die praktische Anwendung der Zufallskonzeptionen.

Eine wirkliche Revolution in Hinsicht auf das Verständnis des Begriffes Zufallsauswahl brachte ein Aufsatz von Jerzy Neyman, der im Jahr 1934 im Journal of the Royal Statistical Society erschien, mit dem Titel: „On the Two Different Aspects of the Representiative Method: The Method of Stratified Sampling and the Method of Purposive Selection“. Entgegen den bisherigen Vorstellungen, dass Zufallsauswahlen ausschließlich unter dem Aspekt gleicher Auswahlwahrscheinlichkeiten zu betrachten seien, entwickelte Neyman in seinem Aufsatz eine Konzeption mit ungleichen Auswahlwahrscheinlichkeiten. Neyman wies darin nach, dass bei einer geschichteten Zufallsauswahl eine höhere Genauigkeit der Stichprobenergebnisse erreicht werden kann, wenn ein überproportional großer Teil des Gesamtstichprobenumfanges auf jene Schichten entfällt, in denen das Untersuchungsmerkmal stärker als in anderen streut.

Dieser Ansatz war indes in Osteuropa nicht neu. Im großen zaristischen Russland waren in der zweiten Hälfte des 19. Jahrhunderts, schon bevor Kiaer seine Arbeiten auf diesem Gebiet begann, ähnliche Konzeptionen angewendet worden (vgl. Zarkovich, 1956, S. 482). Auch dort war ab Beginn des 20. Jahrhunderts intensiv theoretisch auf dem Gebiet der Stichprobenverfahren gearbeitet worden. Die dabei gewonnenen Erkenntnisse fasste A.J. Kowalsky 1924 zusammen. In einem Kapitel über geschichtete Zufallsauswahlen beschrieb Kowalsky genau jene optimale Allokation des Stichprobenumfanges auf die einzelnen Schichten, die - wahrscheinlich unabhängig davon - schon im Jahr 1923 auch Alexander A. Tschuprow und 1934 eben Neyman veröffentlicht haben (vgl. Zarkovich, 1962, S. 487).

Ein zweiter wesentlicher Punkt der Ausführungen Neymans war die Kritik an bewussten Auswahlen. Er wies nach, dass diese Methode für die fehlerhaften Ergebnisse einer von den Statistikern Gini und Galvani erhobenen Stichprobe aus den italienischen Volkszählungsdaten verantwortlich war und stellte ihr die Methode einer geschichteten Zufallsauswahl von Gruppen von Untersuchungsobjekten gegenüber. Weiters wies Neyman in seinem Aufsatz auf die fundamentale Bedeutung der von Fisher entwickelten Schätzkonzeptionen für die Stichprobentheorie hin (vgl. Neyman, 1934, S. 126ff).

Die Verbreitung der Ideen Neymans wurde durch die Einladung des amtlichen Statistikers W. Edward Deming an Neyman, Gastvorlesungen in Washington zu halten, wesentlich beschleunigt. Während eines seiner Vorträge im Jahr 1937 regte 
ihn eine Frage aus dem Publikum zur Entwicklung eines zweiphasigen Auswahlplanes und zur Einführung des Gebrauches von Kostenfunktionen in der Stichprobentheorie an (vgl. Neyman, 1938). Nicht zuletzt wegen der Impulse Neymans verlegte sich in den darauffolgenden Jahren der Schwerpunkt der wissenschaftlichen Beschäftigung mit der Stichprobentheorie von Europa in die Vereinigten Staaten.

Das Aufkommen der kommerziellen Markt- und Meinungsforschung eröffnete in den U.S.A. auch vollkommen neue Betätigungsfelder für Stichprobenerhebungen. Der Ursprung der Markt- und Meinungsforschung geht auf die schon im Abschnitt I.2 erwähnten Probeabstimmungen zum Ausgang von Wahlen zurück, die in den U.S.A. bereits in der ersten Hälfte des 19. Jahrhunderts durchgeführt wurden. Als besonders zuverlässig galten die von der Zeitschrift Literary Digest seit 1916 organisierten Probeabstimmungen zu den Präsidentschaftswahlen, bei der Stimmzettel an eine große Menge von Personen verschickt wurden, deren Adressen aus Telefonverzeichnissen und Verzeichnissen von Automobilbesitzern stammten (vgl. Bortz und Döring, 1995, S. 372; Koschnick, 1995, S. 668f).

Im Jahr 1936 wurden aus diesem Anlass sogar zehn Millionen Stimmzettel ausgesendet. Die Rücklaufquote betrug ca. $24 \%$. Der damit erreichte Teil der gesamten Wählerschaft stimmte mit großer Mehrheit für den republikanischen Kandidaten Alfred Landon. Der Markt- und Meinungsforscher George H. Gallup jedoch entwickelte gemeinsam mit seinen Berufskollegen P. Cherington, Elmo Roper und Archibald M. Crossley Mitte der Dreißiger Jahre eine Stichprobenmethode, die ihnen befriedigender schien als eine solche Auswahl: das Quotenverfahren. Gallup befragte damit lediglich etwa 6.000 Personen und prognostizierte einen Wahlsieg des amtierenden Präsidenten Franklin D. Roosevelt. Roosevelt gewann die Wahl mit einem Stimmenanteil von $62 \%$.

Dieser Prognoseerfolg markiert den Startschuss für die moderne Markt- und Meinungsforschung. Das billigere Quotenverfahren hatte sich, weil es sensationellen Erfolg hatte, als Stichprobenverfahren bewährt, ohne theoretisch fundiert zu sein. Es etablierte sich danach sofort als das bevorzugte Stichprobenverfahren der amerikanischen kommerziellen Markt- und Meinungsforschung. Das Literary Digest hingegen stellte nach diesem Fiasko seine Prognosen ein. Zwei wesentliche Fehlerquellen trugen die Hauptschuld an der hinsichtlich der Kandidatenpräferenz offenbar nicht repräsentativen Literary-Digest-Stichprobe: In der zur Verfügung stehenden Grundgesamtheit wurden die höheren Einkommensschichten im Vergleich zur Grundgesamtheit der Wahlberechtigten deutlich überrepräsentiert. Und die niedrige Rücklaufquote ging noch einmal auf Kosten der unteren Schichten, die Roosevelts Politik des „New Deal" befürworteten.

In Folge des großen Wahlprognoseerfolges erlebten die amerikanischen Umfrageinstitute einen rasanten Aufstieg. Neben den Unternehmen bediente sich nun auch die Politik der Umfrageforschung. Roosevelt etwa benutzte die Meinungsforschung als Quelle der Information über die Zustimmung zu seiner Außenpolitik und zu den Entscheidungen im 2. Weltkrieg. Die erste akademische Umfrageorganisation wurde 1941 an der Universität in Denver mit dem „National Opinion Research Center (NORC)" gegründet (vgl. Kotz und Johnson, 1985, S. 354).

Erst eine Fehlprognose des Ausgangs der Präsidentschaftswahlen 1948 in Folge 
eines nicht mehr erfassten Meinungsumschwunges kurz vor der Wahl, der zu einem knappen Wahlsieg des amtierenden Präsidenten Harry Truman gegen seinen Herausforderer Thomas Dewey führte, beendete die über ein Jahrzehnt andauernde, allzu unreflektierte und sorglose Anwendung des neuen Stichprobenverfahrens. Und dies, obwohl der absolute Fehler des Umfrageergebnisses deutlich geringer ausfiel als 12 Jahre zuvor (vgl. ebd., S. 354). Daraufhin wurden empirische Untersuchungen in Amerika und Europa veranlasst, welche die Qualität der mit dem Quotenverfahren produzierten Umfrageergebnisse überprüften.

Während sich anfangs der Vierziger Jahre die Markt- und Meinungsforschung zu etablieren begann, wurden im Rahmen der Stichprobentheorie einige Ideen Neymans weiterentwickelt: Die Amerikaner Morris H. Hansen und William N. Hurwitz entwickelten das Konzept des Klumpenverfahrens weiter bis zur mehrstufigen Auswahl, die vor allem in der Qualitätskontrolle und auch überall dort Anwendung findet, wo kein Verzeichnis über die einzelnen Elemente der Grundgesamtheit vorhanden ist, und sie verwendeten erstmals den Begriff „Cluster“ dafür (vgl. Sukhatme, 1965, S. $374)$.

Hansen und Hurwitz bauten 1943 auch ein anderes Konzept Neymans weiter aus: Nachdem dieser geschichtete Zufallsauswahlen mit ungleichen Auswahlwahrscheinlichkeiten für Untersuchungsobjekte verschiedener Schichten entworfen hatte, entwickelten Hansen und Hurwitz ein Verfahren mit ungleichen Auswahlwahrscheinlichkeiten innerhalb der Schichten und den dafür benötigten unverzerrten Schätzer. D.G. Horvitz und D.J. Thompson fügten dazu 10 Jahre später auch die Theorie für Ziehen ohne Zurücklegen und den dazugehörenden Schätzer hinzu.

Vorkenntnisse über Grundgesamtheiten wurden in den Dreißiger Jahren neben ihrer Anwendung zur Schichtenbildung für geschichtete Zufallsauswahlen auch zur Verhältnis- und Regressionsschätzung verwendet. 1942 wurden diese Methoden von W.G. Cochran umfassend dargestellt. Ein Jahrzehnt später wurde mit der Differenzenschätzung eine weitere Alternative für die Verwendung von Vorkenntnissen bereitgestellt (vgl. Smith, 1976, S. 186).

Die Arbeit von W. Edward Deming (1944) brachte einen neuen, der Anwendung der Stichprobenmethoden in der Umfrageforschung entstammenden Aspekt in die Stichprobentheorie ein. Der amerikanische amtliche Statistiker beschrieb darin insgesamt 13 Faktoren, welche die Aussagekraft von Umfrageergebnissen beeinträchtigen und wies damit auf die Wertlosigkeit hin, ausschließlich den Stichprobenfehler, also nur eine dieser möglichen Fehlerquellen, kontrollieren zu wollen. Um mit dem Stichprobenfehler die Ungenauigkeit des Stichprobenergebnisses ausreichend beschreiben zu können, muss - im Gegenteil - notwendigerweise auf das tatsächliche Fernbleiben von "Nichtstichprobenfehlern" bei der Planung und Durchführung von Umfragen besonders geachtet werden (vgl. ebd., S. 359). Zu diesen Nichtstichprobenfehlern zählte Deming u.a. Fehler durch das Interviewerverhalten, Fehler im Fragebogen, Fehler, die durch die Teilnahmeverweigerung der Zielpersonen bzw. deren Nichterreichbarkeit entstehen oder Auswertungsfehler.

Damit wurde auch der Nichtstichprobenfehler ein Untersuchungsgegenstand der Stichprobentheorie: Mit dem Fehler durch Nichterreichen bzw. Antwortverweigerung der Befragten setzten sich in der Folge z.B. Hansen und Hurwitz oder auch A. Politz 
und W. Simmons auseinander und entwickelten Schätzer, die diesen Fehler kompensieren können. Deming selbst setzte empirisch die optimale Anzahl von Kontaktierungsversuchen für Befragungen von Angesicht zu Angesicht fest, um die Erreichbarkeit der Zielpersonen zu erhöhen. M.A. El-Badry schlug Mitte der Fünfziger Jahre mehrere Befragungswellen für briefliche Umfragen vor (vgl. Sukhatme, 1965, S. 388f). Die Nichtstichprobenfehler sind gegenwärtig hauptsächlich Forschungsgegenstand der Anwender der statistischen Methoden in der empirischen Sozialforschung.

Ende der Vierziger, Anfang der Fünfziger Jahre schließlich war die Zeit für die Erstauflagen von Büchern gekommen, die sich ausschließlich der nun im Großen und Ganzen voll entwickelten Stichprobentheorie widmeten: Zu diesen „Klassikern“ gehören u.a. Yates (1949), Deming (1950), Cochran (1953), Hansen et al. (1953). Im deutschsprachigen Raum ist in diesem Zusammenhang Kellerer (1953) zu nennen.

In Deutschland und Österreich hatte sich vor dem 2. Weltkrieg (wie auch in anderen europäischen Staaten) ebenfalls bereits ,eine betriebswirtschaftlichen Zwecken verpflichtete Marktforschung ausgebildet“ (Scheuch, 1999, S. 7). Der österreichische Vorläufer der empirischen Sozialforschung Paul F. Lazarsfeld stellte Ende der Dreißiger Jahre die Paneluntersuchung als neues Erhebungsinstrument der Umfrageforschung vor. Dieses kommerziell erfolgreichste Anwendungsgebiet der Stichprobentheorie erhielt nach dem 2. Weltkrieg in Deutschland und Österreich starke Impulse durch die amerikanische Besatzung. Zu den ersten Gründungen zu dieser Zeit zählten auf deutscher Seite das EMNID-Institut und das Institut für Demoskopie in Allensbach, auf österreichischer das österreichische Gallup-Institut und das Institut von Dr. Fessel (vgl. Kapferer, 1994, S. 18ff und S. 101f).

Der Markt für die Markt- und Meinungsforschung hat sich seither enorm entwickelt. Im Jahr 1999 betrug lt. Angaben des „Arbeitskreises Deutscher Marktund Sozialforschungsinstitute e.V. (ADM)" der weltweite Umsatz der Markt- und Meinungsforschungsbranche ca. 13,7 Milliarden EURO, wovon $42 \%$ auf europäische und $37 \%$ auf amerikanische Institute entfielen (Internet: www.adm-ev.de; Zahlen über den Markt für Marktforschung, Ausgabe 2/2001). Den Großteil (etwa zwei Drittel) des europäischen Umsatzes in der Höhe von 5,8 Milliarden EURO teilten sich Großbritannien, Deutschland und Frankreich, wobei dieser Umsatz zuletzt (von 1993 bis 1999) eine durchschnittliche jährliche Steigerungsrate von knapp $12 \%$ aufwies.

In Deutschland hat sich der Umsatz in diesem Zeitraum ebenfalls fast verdoppelt und die Zahl der Institute hat sich um knapp $38 \%$, die Zahl der festangestellten Mitarbeiter um fast $50 \%$ erhöht. Die wichtigsten Auftraggeber kommen dabei aus dem Bereich der Konsum- und Gebrauchsgüterindustrie, die 2000 z.B. 51 \% des Umsatzes der im ADM zusammengefassten Institute einbrachten. Medien und Verlage waren daran mit $14 \%$ daran beteiligt, während öffentliche Auftraggeber nur mehr $2 \%$ des jährlichen Umsatzes beisteuerten.

In den letzten Jahrzehnten ergaben sich gerade in dem angesprochenen Anwendungsbereich der Stichprobentheorie eine Reihe von Fragestellungen, die bis zur Gegenwart die Stichprobentheoretiker und -praktiker beschäftigen. Als Beispiele seien aus dem deutschsprachigen Raum genannt: die Entwicklung und kritische Beurteilung von Verfahren, welche die Daten zweier Stichproben zu einer einzigen fusionieren (vgl. etwa Rässler und Fleischer, 1997), die Entwicklung von sogenannten 
Imputationstechniken, welche die in einer Stichprobe bei einzelnen Untersuchungsobjekten fehlenden Daten durch bei anderen Untersuchungsobjekten vorhandene ergänzen (vgl. etwa Rässler, 2000), die Untersuchung der Verwendbarkeit des Internets als Datenerhebungsinstrument (vgl. etwa Bandilla und Hauptmanns, 1998) und die Erstellung von Stichprobendesigns für telefonische Befragungen am sich laufend verändernden Telefonsektor (vgl. etwa Gabler und Häder, 1999). Auch die theoretische Untersuchung der Quotenverfahren (Quatember, 1996 und 1997) gehört zu diesen durch die Praxis der Markt- und Meinungsforschung motivierten Forschungsgebieten der Stichprobentheorie.

Die rasante Entwicklung immer leistungsfähigerer Computer förderte in den Siebziger Jahren das Interesse für „Resampling-Verfahren“ wie die Jackknife-Schätzung, mit deren Hilfe allein durch Verwendung der in einer Stichprobe erhobenen Daten die statistischen Eigenschaften von Schätzern - z.B. wenn sie aus komplexen Stichproben stammen - untersucht werden können. Bradley Efron, Statistiker an der kalifornischen Stanford Universität, veröffentlichte im Jahr 1979 seine Idee für ein weiteres solches computerintensives Verfahren: die Bootstrap-Methode (vgl. Efron, 1979). Die daraufhin einsetzende formale Entwicklung seiner Vorstellungen benötigte fast ein Jahrzehnt. Diese theoretischen Untersuchungen haben in der Folge die Konsistenz der Bootstrap-Schätzer bewiesen und ihre asymptotische Verteilung für eine Vielfalt interessierender Modelle abgeleitet. Es wurde ferner erörtert, in welchen Situationen das Bootstrapverfahren keine vertrauenswürdigen Schätzer hervorbringt und dass es vielfältiger anwendbar und zuverlässiger als die Jackknife-Methode ist (vgl. Beran, 1992, S. 565). Särndal et al. (1992) fassten diese und andere neue Entwicklungen im Zusammenhang mit komplexen Stichproben zusammen.

Schließlich sind aus dem deutschsprachigen Raum in den letzten Jahren einige Arbeiten zu nennen, welche diese aktuellen Entwicklungen in der Stichprobentheorie noch abrunden. Dazu gehören u.a. Arbeiten aus dem Bereich der Messproblematik wie z.B. die Betrachtung der Unschärfe von beobachteten Daten (vgl. etwa Viertl, 1996) und der Aufbau einer Stichprobentheorie unter Berücksichtigung von Fehlklassifikationen beim Messvorgang (vgl. Wagner, 2001).

\section{Schlussbemerkungen}

In den für die Stichprobentheorie stürmischen Dreißiger Jahren des 20. Jahrhunderts begann Sukhatme (1935) seinen Aufsatz mit dem Titel „Contribution to the Theory of the Representative Method“ im Journal of the Royal Statistical Society mit der Feststellung: „In social research it is often required to estimate the average value of a character of some individuals. Until recently such averages have been calculated only from the data of general censuses" (ebd., S. 253). Weniger als sieben Jahrzehnte später blicken wir auf eine vollständig entwickelte Theorie (und Praxis) der Stichprobenmethode, die allgemein akzeptiert ist, so dass sich dafür immer neue Anwendungsgebiete auf kommerzieller wie auch auf akademischer Ebene auftun. $\mathrm{Zu}$

Recht darf man deshalb wohl vom 20. Jahrhundert ohne Übertreibung auch als dem Jahrhundert der Stichproben sprechen. 
A. Quatember

Literatur 
Author's address:

Dr. Andreas Quatember

Johannes-Kepler-Universität Linz

IFAS - Institut für Angewandte Statistik

Altenberger Straße 69

A-4040 Linz

Austria

Tel. $+437322468 / 8267$

Fax +43732 2468/9846

Email: andreas.quatember@jk.uni-linz.ac.at 